\title{
CHARACTERIZATION OF ANCIENT TRIPITAKA
}

\author{
Y. X. Gong ${ }^{\mathrm{a}}$, L. Geng ${ }^{\mathrm{b}}$, D. C. Gong ${ }^{\mathrm{c}}$ \\ ${ }^{a}$ PhD Student, Faculty of History and Archaeology of Science and Technology, University of Science and Technology of China, \\ Hefei, P. R. China - gongyuxuan@ hotmail.com \\ ${ }^{\mathrm{b}} \mathrm{PhD}$ Student, Faculty of History and Archaeology of Science and Technology, University of Science and Technology of China, \\ Hefei, P. R. China - genglu@ mail.ustc.edu.cn \\ ${ }^{\mathrm{c}}$ Professor, Faculty of History and Archaeology of Science and Technology, University of Science and Technology of China, Hefei, P. \\ R. China-gdclucky@ustc.edu.cn
}

KEY WORDS: Tripitaka, Fiber Analysis, Thin-Layer Chromatography, Indigo

\begin{abstract}
:
Tripitaka is the world's most comprehensive version of Buddhist sutra. There are limited numbers of Tripitaka currently preserved, most of them present various patterns of degradation. As little is known about the materials and crafts used in Tripitaka, it appeared necessary to identify them, and to further define adapted conservation treatment. In this work, a study concerning the paper source and dyestuff of the Tripitaka from approximate $16^{\text {th }}$ century was carried out using fiber analysis and thin-layer chromatography (TLC). The results proved that the papers were mainly made from hemp or bark of mulberry tree, and indigo was used for colorizing the paper. At the end, we provide with suggestions for protecting and restoring the ancient Tripitaka.
\end{abstract}

\section{INTRODUCTION}

The term Tripitaka which derives from Tang dynasty, is originally defined as the name of the complete set of sutra collections. The existing copies cover a wild range of languages, including Chinese, Tibetan, Mongolian, Manchu, Tangut, Japanese, Pali, Dai and English. Wuwei Museum of Gansu preserves Tripitaka over 40,000 volumes including 5317 volumes of Tibetan Tripitaka dated from Ming Dynasty and 6,300 copies in Chinese, which hold two thirds of its museum collections (Miao, 2005). Among these, Tibetan Tripitaka which known as "a Treasure of the World" was first complied by Tibetan Buddhists in $14^{\text {th }}$ century. It is of important historical, scientific and artistic interests. The Tibetan Tripitaka are handwritten or printed on Tibetan paper, a high quality paper made with mainly bark of mulberry tree in Lintao, Gansu. The paper is hard to damage, owing to the feature of thick and solid, and soft and smooth make it easy to paint. Having experienced of thousands years, most of the Tripitaka didn't show any signs of aging (Gao, 2012) until the serious flood which happened in 1975. Because of the poor preservation conditions and lack of restorer, a large number of Tripitaka were flooded and seriously damaged. Some of these Tripitaka present nowadays various patterns of degradation, e.g. cracks, folds and traces of moulds. In order to define preservation conditions and adapted conservation treatments, a study concerning the source and dyestuff of the paper and paper-making technique was carried out in this paper, using fiber analysis and thin-layer chromatography. The conservation treatment and preservation conditions are also discussed here.

\section{MATERIALS AND METHODS}

\subsection{Materials}

Samples were selected from the Tibetan Tripitaka of Wuwei, they are identified as W1, W2, W3 and W4. Table 1 shows the information of the samples. 
The International Archives of the Photogrammetry, Remote Sensing and Spatial Information Sciences, Volume XL-5/W7, 2015 25th International CIPA Symposium 2015, 31 August - 04 September 2015, Taipei, Taiwan

Table 1 Information of the samples

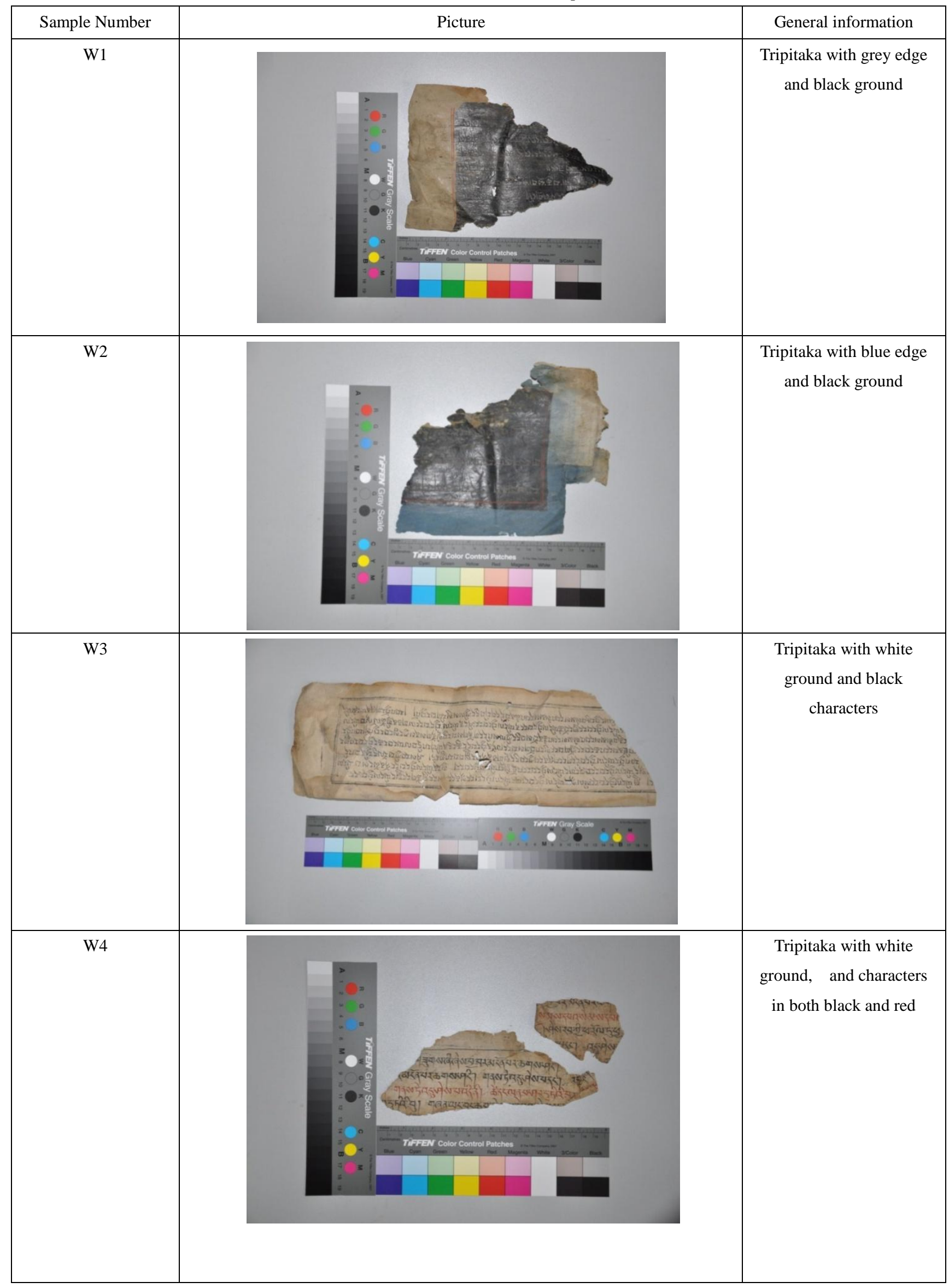




\subsection{Methods}

a. Analysis of the craft

The paper-making technique and writing mode were defined by observing the characterization of the paper and the characters.

\section{b. Identification of the paper material}

Small pieces were taken from W1, placed in clean test tube and immersed with purified water. The paper was then dispersed completely into fiber through vibration. A small volume of the fiber was picked up and placed onto the glass slide using tweezers. The fiber was then dyed with two drops of stain (Iodine - zinc chloride), and covered with cover slip. The sample preparation procedure was repeated for W2, W3 and W4. Fiber analysis was carried out with a XWY-VI device to observe the morphology.

\section{c. Analysis of the blue dye}

TLC was applied in the analysis of the dye. A small sample was taken from the blue area of W2, and soaked in acetic acid at room temperature for dye extraction. The natural dye of indigo which extracted from bluegrass was purchased from Guizhou, China, and selected as the control sample. The solution was configured by immersing a small amount of the indigo in a defined amount of acetic acid. A silica gel plate was measured and marked with $1 \mathrm{~cm}$ line and $7 \mathrm{~cm}$ line. Two small dots were drawn at the $1 \mathrm{~cm}$ line with the solution by using a pipette. The

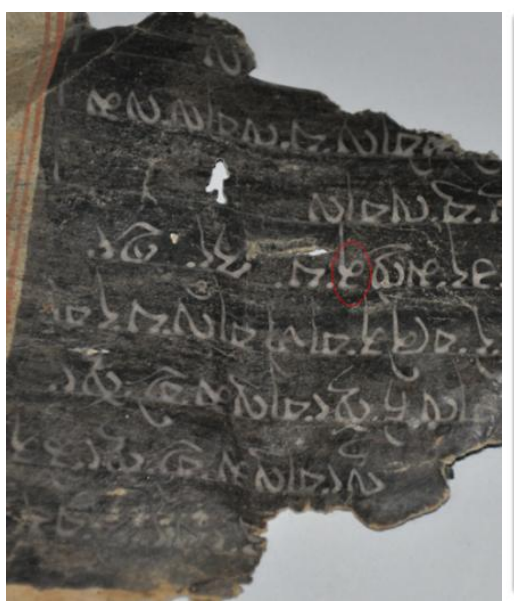

Fig. 1 Sample W1 silica gel plate was then placed into the developing solvent of a 8:1:1 mixture of benzene, acetone and nitrobenzene and the beaker was sealed with plastic wrap. The silica gel plate was recovered after standing for approximate 30 to 40 mins, till the developing solvent reached the $7 \mathrm{~cm}$ line (Kharbade, 1988). This procedure was repeated for the blue dye extracted from W2.

\section{RESULTS AND DISCUSSIONS}

\subsection{Analysis of the craft}

Obviously, no curtain texture was observed on W1, W2, W3 and W4. The visible linen texture was found on W3, which may result from the paper-making technique of pouring. The paper of W1 and W2 are a bit thicker and can be detached into three layers, whereas, W3 and W4 only have two layers. As shown in Fig. 2, clear "hidden lines" were observed on W1 and W2, which were used as columns for regulating the characters. The same characters on W1, W2 and W4 were written in slightly different styles with flexible strokes and in uneven size (see Fig. 1 and 4). Therefore, we believe that W1, W2 and W4 were hand-written by using hard-tipped pen, since no sign of roughness (usually resulted from brush pen writing) was found. While W3 was made by block printing, as the characters were aligned regularly, and showing in exactly same mode and size (see Fig. 3).

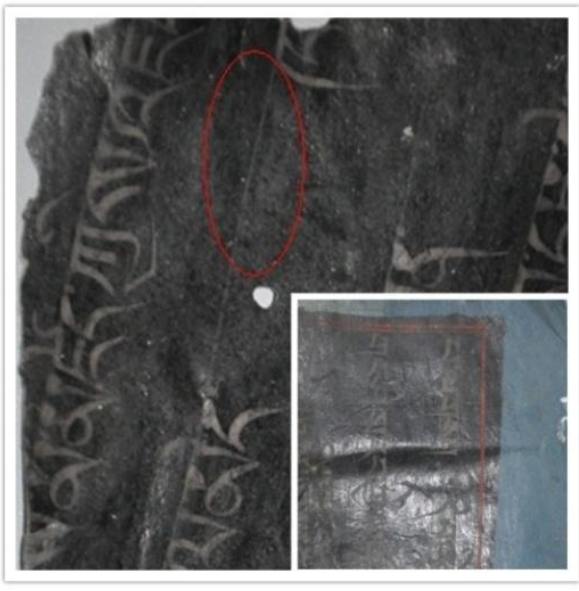

Fig. 2 Sample W2 


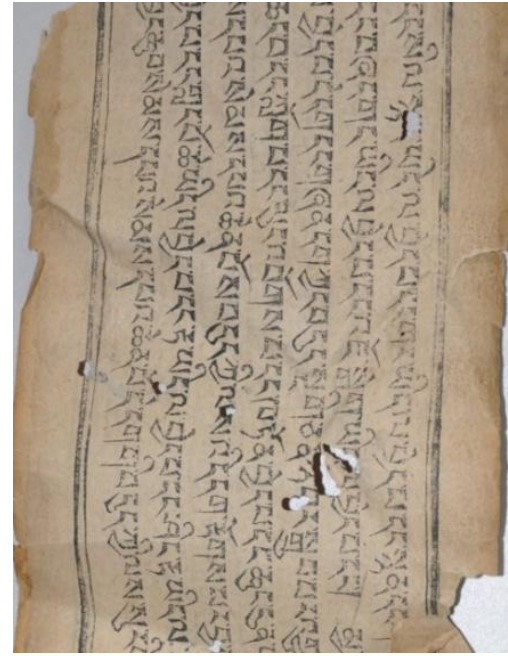

Fig. 3 Sample W3

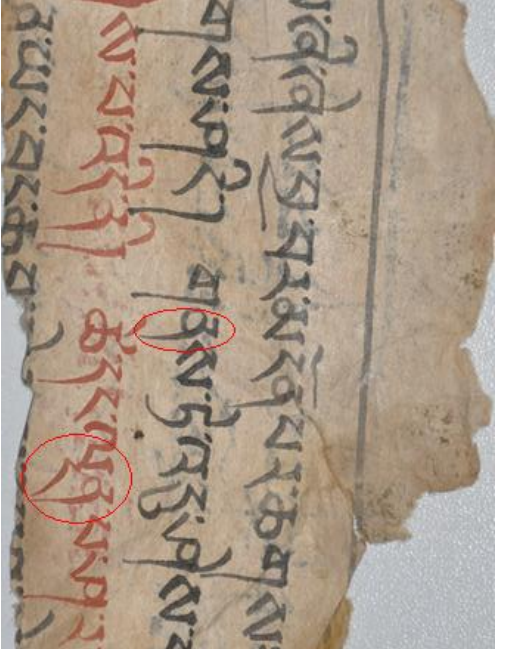

Fig. 4 Sample W4

\subsection{Identification of the papers' fiber}
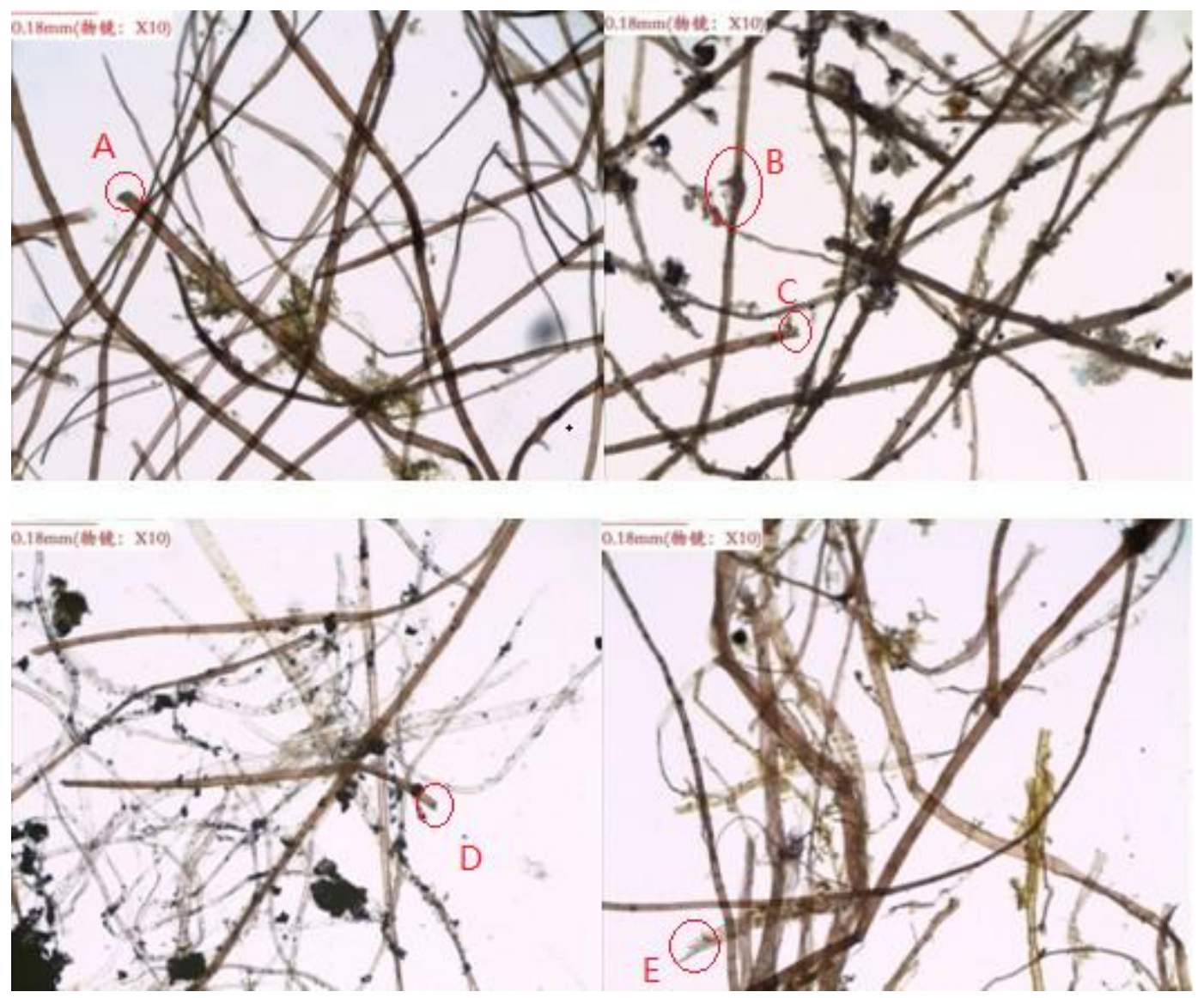

Fig. 5 Morphology of the fiber (from up left to bottom right areW1, W2, W3, W4)

As shown in Fig. 5, pure and long fiber without any non-fiber cell was observed on W1 and W2, the mean width of the fiber was measured at $17-19 \mu \mathrm{m}$. After dyeing, the fiber presented a reddish brown surface. The cross-section texture was found on the sheer film coated fiber wall. In addition, the traces of shear (A and C) and pounding (B) were observed which pointed to the steps of fiber cutting and pounding during paper-making process. It further evidenced the mature paper-making technology in Ming dynasty. As a result, the fiber was identified as the bark of mulberry tree (Gong, 2014).

Two different types of fiber were discriminated on the fiber morphology of W3. One was from the bark of mulberry tree which has the same characteristics as W1 and W2. Another 
fiber was thin, stiff and of $8 \mu \mathrm{m}$ width, very few bending was found. These characterizations match with the bamboo fiber. Moreover, starch was used as the sizing agent during paper-making, which resulted in massive blue substances after dyeing, and similar trace of shear (D) was found.

Fig. 5 shows also two different kinds of fiber for W4. The majority was pure and with $21 \mu \mathrm{m}$ width which showed reddish brown after dyeing. The vertical grains were visible. Hence the material was identified as the fiber of hemp. The appearance of fibrillation (E) was observed, which resulted from completely pounding the fiber, to ensure the softness of the finished paper. It is a key step for yielding high quality paper (Gong, 2014). Another type of fiber was thin, fascicular and showed yellow tint after dyeing. The mean width was $15 \mu \mathrm{m}$, which could be the jute fiber.

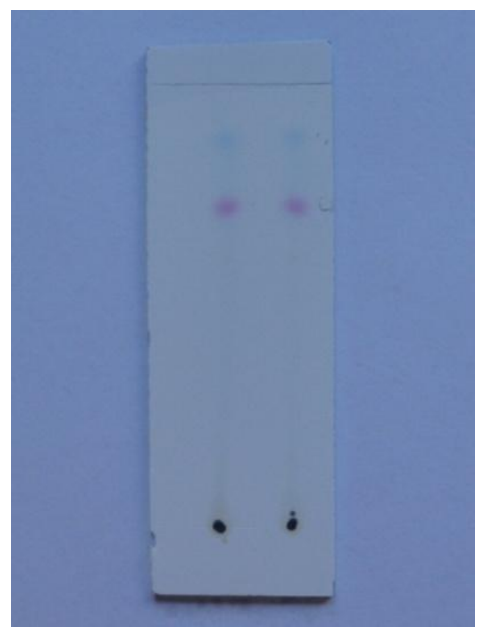

\subsection{Thin-layer chromatography analysis of the blue dye}

It was known that indigotin and indirubin were the two major coloring matters contained in the control sample, which represented by the blue dot and red dot (Chen, 2000) respectively on Fig. 6. The TLC results are shown in Table 2. The same $\mathbf{R}_{f 1}$ value confirmed the existence of similar indigotin in both of the samples (Tayade, 2014), whereas the slight different $\mathrm{R}_{f 2}$ value indicated the diversity of indirubin (Kharbade, 1988). The difference was probably resulted from the different techniques used in extracting natural dye from plant during ancient period and modern times (Zhang, 2010). In conclusion, the dye used to colorizing W2 was identified as indigo, which contains similar indigotin as the modern sample. As recorded by historical document, indigo was the most popular blue dye that widely used in Ming dynasty (Tan, 2011).

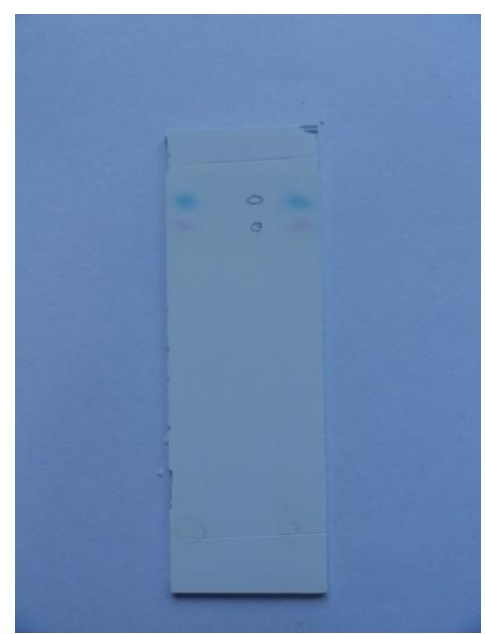

Fig. 6 Silica gel plate recovered from developing solvent (left: control sample, right: W2)

\begin{tabular}{|l|c|c|c|c|}
\hline \multicolumn{1}{|c|}{ Samples } & $\begin{array}{c}\text { Measurement of blue } \\
\operatorname{dot}(\mathbf{c m})\end{array}$ & $\mathbf{R}_{f 1}$ value & $\begin{array}{c}\text { Measurement of red } \\
\operatorname{dot}(\mathbf{c m})\end{array}$ & $\mathbf{R}_{f 2}$ value \\
\hline W2 & 5.3 & 0.88 & 5 & 0.83 \\
\hline control sample & 5.3 & 0.88 & 4.7 & 0.73 \\
\hline
\end{tabular}

Table 2 TLC results

\section{SUGGESTIONS ON PRESERVING THE TRIPITAKA OF WUWEI}

Based on the above analysis, we provide with the following suggestions on preserving and restoring the Tripitaka:

a. Use the suitable material to patch the paper

The papers of W1 and W2 were made from the bark of mulberry tree. W3's paper was made from both the bark of mulberry tree and bamboo fiber. The paper material of W4 was the mixture of hemp and jute fiber. During the process of restoring and reinforcing the Tripitaka, it is suggested to use the paper with similar material.

b. Prevent the Tripitaka from the damages of dust and mould Paper culture relics usually suffer from the damages of mould and dust during long-term preservation. Paper is easy to be 
attached with dust, and gives rise to the friction during turn over of the page, therefore, results in fluffiness and perforation. The cellulose of paper fiber would be degraded and corroded by microbe and metal ion attached to the dust $(\mathrm{Li}, 2011)$, as a result, reduce the strength of the paper (Hua, 2013). In fact, adhesion and mildew are already found on the Tibetan Tripitaka due to flooded, therefore, it is necessary to carry out feasible actions on preventing the Tripitaka from the damages of dust and mould, e.g. place Tripitaka into box made with non-harmful materials, or cover the loose page or the fragment with rice paper.

c. Use the appropriate dyestuff in restoration

Indigo with the components of indigotin and indirubin was used in the colorization of W2's paper. Therefore, to recover the original face of the Tripitaka, the appropriate dye should be applied in restoration.

\section{CONCLUSIONS}

Being a part of the paper culture relics, Tripitaka carries the precious and valuable records of human civilization and the development of Buddhism. Although the writing language on Tripitaka and its translation was largely investigated, very little was known about the materials and techniques used. As the number of Tripitaka reduced over the years, it is time-critical to take necessary actions to prevent the treasure from damage. This analytical research employed scientific approaches to study the crafts and materials used. It demonstrated that the papers were made mainly from the bark of mulberry tree, bamboo, hemp and jute. The use of indigo as the dye to colorize the paper is also highlighted here. This research demonstrates evidence of defining adopted treatment, and also provides inspiration on studying the history of science and civilization of ancient Tibet.

\section{REFERENCES}

Chen, Y. S., Xie, Y. L., Xiong, Y. F., Jia, Y. Y., Huang, X. J., 2000. The identification of blue - green dye on wool textiles from Shanpula tombs in Xinjiang. Sciences of conservation and archaeology, 12 (1), pp. 15-21.

Gao, H., 2012. Ancient Books Collection of Wuwei Museum and its conservation status. Cultural Heritage of Longyou, 36 (2), pp. 86-88.

Gong, D. C., Yang, H. Y., Li, X. C., 2014. Studies on the papermaking techniques and compositions of fillers of the Xuanquanzhi paper in Dunhuang, Gansu province. Cultural Relics, 700 (9), pp. 85-90.

Hua, X. L., 2013. Suggestions on the Conservation of Paper Cultural Relics. Article World, 2 (4), pp. 46.

Kharbade, B. V., Agrawal, O. P., 1988. Analysis of Natural Dyes in Indian Historic Textiles. Studies in Conservation, 33 (1), pp. 1-8.

Li, P. X., 2011. Briefly Dicussion of the Damages and Conservation of Paper Cultural Relics. Silk Road, 217 (24), pp. 96-97.

Miao, Z., 2005. Buddhist Tripitaka of China. Buddhism of Guangdong, (1), pp. 4-13.

Tan, G. W., 2011. Plant dyes in ancient China. Cartography, (6), pp. 64-71.

Tayade, P. B., Adivarekar, R. V., 2014. Extraction of Indigo dye from Couroupita guianensis and its application on cotton fabric. Fashion and Textiles, 10, pp. 1-16.

Zhang, X. M., Wei, X. N., Lei, Y., Cheng, X. L., Zhou, Y., 2010. Micro and Nondestructive Analysis of Blue Dyes from Silk Fabrics Decorative Painting of Historic Building. Spectroscopy and Spectral Analysis, 30 (12), pp. 3254-3257. 